\title{
Morphology and structure of nearby starburst galaxies in the UV and optical
}

\author{
Christopher J. Conselice, John S. Gallagher, Nicole L. Homeier \\ Astronomy Department, University of Wisconsin-Madison, \\ Madison, WI 53706, USA \\ Daniela Calzetti, and Anne L. Kinney \\ Space Telescope Science Institute, Baltimore, MD 21218, USA
}

\begin{abstract}
We present results of a panchromatic morphological study to determine how UV and $\mathrm{H} \alpha$ morphology relates to the underlying stellar populations, the ISM, and structure of nearby starbursts.
\end{abstract}

\section{Introduction}

By studying the distribution of light in starburst galaxies at different wavelengths, including $\mathrm{H} \alpha$ and the UV, we can better understand the mechanisms that drive star formation processes. Starburst galaxies have also recently received a boost of attention due to their similarities with high-redshift galaxies, and the possibility exists of using local starbursts to determine the features of distant galaxies. By doing this, we are beginning to gain insight into the mechanisms by which a significant fraction of galaxies have probably evolved. In addition, by studying the distribution of $\mathrm{H}$ II regions and how they correspond, or do not correspond to bright UV starbursting regions, we can determine how useful UV fluxes are for measurements of star formation in distant galaxies.

We have investigated using both ground based WIYN 3.5m telescope $B, R$ and $\mathrm{H} \alpha$ images and HST-WFPC2 and -FOC UV images, the morphological properties of starbursts to determine how morphology relates to the flux in each waveband. Since each wavelength samples a unique aspect of the stars and gas in our sample, we are able to determine how these various features and mechanisms correlate. By studying how the morphology and relative fluxes of starbursts regions in a starbursts changes as a function of wavelength can give us unique information about the structure and interstellar medium of starbursts, including such things as: dust content, photon leakage, stellar winds, and the relationship between stellar populations and the ISM of a starburst, as well as the ability to date a star forming region. Our sample of galaxies includes NGC 3690, NGC 3351, NGC 3310, and NGC 7673, representing a wide range of starburst galaxy morphologies. By studying the differences between the various wavelengths for each starburst, we are also able to determine how relationships between wavelengths change within various starburst galaxy morphologies. 

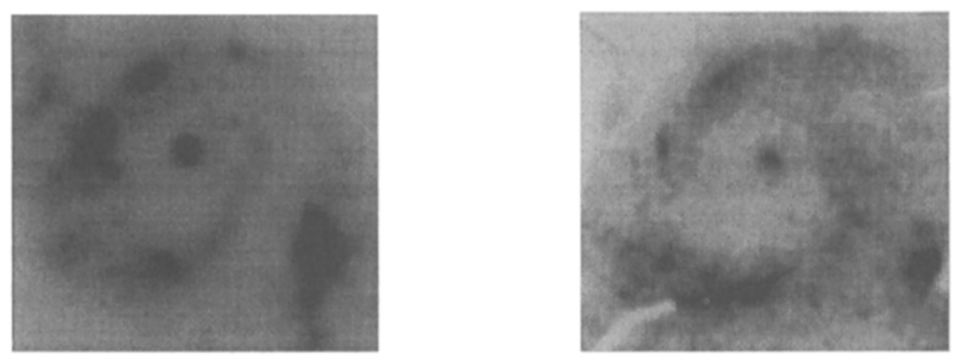

Figure 1. Left: NGC 3310 in $\mathrm{H} \alpha$. Right: NGC 3310 in UV

\section{Data and method}

The data used in this study were taken with the WIYN $3.5 \mathrm{~m}$ telescopes in the $B$ and $R$ filters, and a narrow band $\mathrm{H} \alpha$ filter. The UV images came from the FOC and WFPC2 on the HST. The images were reduced in the usual fashion. The HST images are at different orientations, scales and pixel sizes from the WIYN images. After alignment, we then are able to make color maps, as well as quantify how the relative distribution of light in the star forming regions changes as a function of wavelength.

\section{Results}

By examining the color images of the starbursts used in this study a few preliminary conclusions about the panchromatic light distribution in nearby starbursts can be made.

By comparing the UV to $\mathrm{H} \alpha$ image ratio maps, it is clear that the $\mathrm{H} \alpha$ flux does not always correlate spatially with the UV flux, originating from very young stars. We find in some starbursts areas of enhanced $\mathrm{H} \alpha$ flux where we do not see a significant corresponding UV flux, and we also see the reverse - areas of intense UV flux without corresponding $\mathrm{H} \alpha$ flux. It must be noted however, that most $\mathrm{H}$ II regions have corresponding high UV fluxes, and vice-versa. The areas where $\mathrm{H} \alpha$ and UV light do not correspond are the interesting exceptions, since they may reveal how star formation proceeds throughout the star bursting regions in starburst galaxies. $\mathrm{H}$ II regions with high $\mathrm{H} \alpha$ fluxes, but with no corresponding UV flux are possibly regions where hydrogen gas is ionized by 'leakage' from neighboring starburst regions, star forming regions with a high dust content, or signify an older starburst. Alternatively, areas with high UV fluxes but with no $\mathrm{H} \alpha$ could be areas where the gas surrounding the young stars was expelled by stellar winds, or other high-energy phenomenon such as supernova. How the distribution of UV and $\mathrm{H} \alpha$ light correlates is found to be a function of the morphology of the host starburst galaxy, with more disturbed galaxies showing a larger difference between UV and $\mathrm{H} \alpha$ regions, such as NGC 3690. While galaxies with a more symmetric structure, such as NGC 7673 show a good correspondence between the H II and UV starburst regions. 